\title{
Isolation and characterization of nanocrystalline cellulose from roselle-derived microcrystalline cellulose
}

\begin{abstract}
ABSTRCT
Roselle fiber is a renewable and sustainable agricultural waste enriched with cellulose polysaccharides. The isolation of Nanocrystalline cellulose (NCC) from roselle-derived microcrystalline cellulose (MCC) is an alternative approach to recover the agricultural roselle plant residue. In the present study, acid hydrolysis with different reaction time was carried out to degrade the roselle-derived MCC to form NCC. The characterizations of isolated NCC were conducted through Fourier Transform Infrared Ray (FTIR), Transmission Electron Microscopy (TEM), Field Emission Scanning Electron Microscopy (FESEM), Atomic Force Microscopy (AFM), Dynamic Light Scattering (DLS), Energy Dispersive Spectroscopy (EDS), X-ray Diffraction (XRD), Thermogravimetric Analysis (TGA) and Differential Scanning Calorimetry (DSC). As evaluated from the performed morphological investigations, the needle-like shape NCC nanostructures were observed under TEM and AFM microscopy studies, while irregular rod-like shape of NCC was observed under FESEM analysis. With 60 min hydrolysis time, XRD analysis demonstrated the highest NCC crystallinity degree with $79.5 \%$. In thermal analysis by TGA and DSC, the shorter hydrolysis time tended to produce NCC with higher thermal stability. Thus, the isolated NCC from roselle-derived MCC has high potential to be used in application of pharmaceutical and biomedical fields for nanocomposite fabrication.
\end{abstract}

Keyword: Roselle; Nanocrystalline cellulose; Morphology; Crystallinity; Thermal stability. 